\title{
Precision Interfaces for Different Modalities
}

\author{
Haoci Zhang \\ Columbia University \\ zhanghaoci@gmail.com \\ Thibault Sellam \\ Columbia University \\ tsellam@cs.columbia.edu
}

\author{
Viraj Rai \\ Columbia University \\ vr2376@columbia.edu \\ Eugene $\mathrm{Wu}$ \\ Columbia University \\ ewu@cs.columbia.edu
}

\begin{abstract}
Building interactive tools to support data analysis is hard because it is not always clear what to build and how to build it. To address this problem, we present Precision Interfaces, a semi-automatic system to generate task-specific data analytics interfaces. Precision Interface can turn a log of executed programs into an interface, by identifying micro-variations between the programs and mapping them to interface components. This paper focuses on SQL query logs, but we can generalize the approach to other languages. Our system operates in two steps: it first builds an interaction graph, which describes how the queries can be transformed into each other. Then, it finds a set of UI components that covers a maximal number of transformations. To restrict the domain of changes to be detected, our system uses a domain-specific language, PILang. We describe each of Precision Interface's components, showcase an early prototype on real program logs, and discuss future research opportunities.

This demonstration highlights the potential for data-driven interactive interface mining from query logs. We will first walk participants through the process that Precision Interfaces goes through to generate interactive analysis interfaces from query logs. We will then show the versatility of Precision Interfaces by letting participants choose from multiple different interface modalities, interaction designs, and query logs to generate 2D point-and-click, gestural, and even natural language analysis interfaces for commonly performed analyses.
\end{abstract}

\section{ACM Reference Format:}

Haoci Zhang, Viraj Rai, Thibault Sellam, and Eugene Wu. 2018. Precision Interfaces for Different Modalities. In Proceedings of 2018 International Conference on Management of Data (SIGMOD'18). ACM, New York, NY, USA, 4 pages. https://doi.org/10.1145/3183713.3193570

\section{INTRODUCTION}

Data analysis is a fundamental driver of modern decision making, and interactive interfaces enable users to perform this task without knowing how to program. Indeed, a well-designed interface provides interaction components for the users to easily accomplish

Permission to make digital or hard copies of all or part of this work for personal or classroom use is granted without fee provided that copies are not made or distributed for profit or commercial advantage and that copies bear this notice and the full citation on the first page. Copyrights for components of this work owned by others than ACM must be honored. Abstracting with credit is permitted. To copy otherwise, or republish, to post on servers or to redistribute to lists, requires prior specific permission and/or a fee. Request permissions from permissions@acm.org.

SIGMOD'18, fune 10-15, 2018, Houston, TX, USA

(C) 2018 Association for Computing Machinery.

ACM ISBN 978-1-4503-4703-7/18/06 . .\$15.00

https://doi.org/10.1145/3183713.3193570 their tasks, and hides the technical complexity of the underlying system. For instance, the Google Finance stock visualization incorporates a time-range filter so that users from a broad audience can explore prices over time. The same interface would not satisfy market analysts who want to aggregate sales information and perform roll-ups and drill-downs; for them, a rich interface such as Tableau would be preferable. In a more extreme scenario, a single text box that lets users type a full program would be effective for skilled an engineer who wants to write arbitrary programs. This suggests that the appropriate interface provides interactive controls that easily express operations important to the user's desired analyses.

Unfortunately, it is currently not cost-effective, perhaps even not possible, to create an interactive interface for every possible analysis: there are simply too many use cases, each being unique, and development costs are too high. To illustrate, consider a popular interactive tool such as Tableau. Tableau's designers carefully identified a valuable and commonly repeated set of analytical data operations (for instance, selecting measures, dimensions, aggregates or filters) and mapped them to interface interactions (e.g, drag and drops and clicks on contextual menus). Much resources were needed to 1) identify the salient set of analysis queries and 2) design the interactions to express them, and those resources are not available for every use case. The scientist that repeatedly performs the same set of custom analyses, the rural insurance researcher that must retrain new recruits to tweak queries for each farm to be covered, and the overworked IT staff that performs analyses on behalf of other departments, are left to manually write, edit, manage, and visualize analysis queries on their own.

A number of programming toolkits $[4,12]$ make building interfaces easier, but they neither help identify salient analysis queries nor map them to interface designs. Ideally, interactive analysis interfaces should be generated automatically. To this end, systems to generate forms and interfaces were previously introduced for SQL [6, 7], graph databases [2, 14], natural language interfaces [5] and authors have presented methods to simplify visualization workflows [11]. But a number of key challenges remain to be explored. First, the variety of use cases means that even for the same data set, different users might be interested in different analyses (and consequently, different queries). Second, multiple query languages exist and should be easy to support. Third, interaction modalities are changing quickly - new devices enter the market and interface researchers propose new interactions, taking them into account and choosing the appropriate set of widgets for an analysis is difficult.

Our prior technical report [13] proposed Precision Interfaces, a system that automatically generates interactive interfaces tailored 
to prior analyses by analyzing query logs. We observed that structural differences between pairs of queries (e.g. adding an attribute to the SELECT clause) can be mapped to user interactions in a generated web application (e.g. clicking a button, dragging a label). Therefore, our system iterates through query logs and looks for interesting structural differences between pairs of queries. It then generates an interaction graph, where each vertex corresponds to a query and each edge represents an interaction. To select the most appropriate widgets, it assigns a cost to each widget and treats widget selection as an optimization problem.

To address the first challenge, the system uses query logs as the API for interactive interface generation. Such logs encode the analysis users perform in practice, and are easily accessible as they are often automatically collected by data processing systems by way of provenance capture subsystems, as part of recovery and auditing mechanisms such as DBMS query logs, or by user-facing applications such as Jupyter.

To solve the second challenge, the system models and analyzes queries using their abstract syntax tree representation. It makes basic assumptions such as knowledge of common data types and simple structures such as collections and lists, but otherwise is agnostic to the specific language. We have shown support for SQL and SPARQL query languages (though each log must be languagehomogenous).

To address the third challenge, we decouple query transformations from interaction component specifications using a simple interaction model. This demonstration focuses on showing how this model can be used to support generating interfaces with different input modalities-form-based, gesture-based, and natural-languagebased interfaces. Section 2 introduces the system overview and our interaction model, Section 3 describes how interaction components under different modalities fit within this model, and Section 4 describes our demonstration.

\section{SYSTEM OVERVIEW}

To provide context for the demonstration, this section provides a high-level description of how Precision Interfaces transforms query logs into simple interactive interfaces. We focus on how interactions and interface widgets are modeled and refer the reader to the technical report for a detailed system description [13].

Figure 1 illustrates the workflow. An input sequence of queries is ingested and parsed into a sequence of abstract syntax treesone tree for each query. This transformation enables the system to analyze changes between queries as sets of sub-tree transformations, which can be detected using fast ordered tree-matching algorithms [3], rather than substring replacements, which are sensitive to spacing, naming, and other trivial syntactic differences. In this way, we model an interaction $t_{\pi}(q, \tau)=q^{\prime}$ as a function that replaces the subtree in $q$ rooted at the path $\pi$, with a new subtree $\tau$. Using this definition, we model the query log with an interaction graph, where each query is a node, and edges are defined by the interaction $(\pi, \tau)$ between each pair of queries. The Interaction Miner generates this graph.

This formulation is important, because it serves as the bridge between queries and components of the interactive interface that the user will manipulate. To this end, we model an interactive widget $w=\left(t_{\pi}, \Omega, f\right)$ (e.g., a dropdown list) as subtree generators that translate user inputs into candidate $\tau$ s. It is initialized with a domain of possible values $\Omega$ that the widget can generate (e.g., the range of possible numbers for a slider), an interaction $t_{\pi}$, and a function $f(o \in \Omega)=\tau$ that maps an element in $\Omega$ to a subtree $\tau$. Thus, if $o \in \Omega$ is the current value of the widget, then it can transform the interface's current query $q$ as: $w(q)=t_{\pi}(q, f(o))$.

Example 2.1 (A Simple Dropdown Widget). Consider a query log where the GROUPBY attribute is changed many times; we observe many interactions $t_{\pi}$ that share the same path $\pi$, but their replacement subtrees differ in the attribute's string value. From the query $\log$ or the database, we can identify a set of possible attributes $\Omega$ and initialize a dropdown menu with it. Finally, $f()$ can be defined as generating a replacement subtree that sets the attribute's string value to the currently selected value in the dropdown. Such a dropdown can now be used to change the GROUPBY attribute in the interface's current query $q$.

In this way, the Interaction Mapper models interface generation as an edge cover problem over the interaction graph. A given interactive widget can express a set of edges in the graph, and the goal is to select a set of widgets that form an edge cover while minimizing various interface complexity measures. We use a contraction-based heuristic to solve this problem. We provide a simple filtering language called PILang to filter the interaction graph prior to generating the interface; the technical report describes the language syntax and how the filtering is pushed down into the parser and interaction miner.

\section{MULTI-MODAL INTERACTIONS}

The previous section described how Precision Interfaces models interactions as parameterized subtree transformations, and interface components as subtree generators. This section describes how this abstract model serves as a bridge to add new interface components, and flexibly adapt mined interfaces to support different input modalities such as form-based, gestural, and natural language interfaces.

Consider the following trivial example:

Example 3.1. There are two queries in the log.

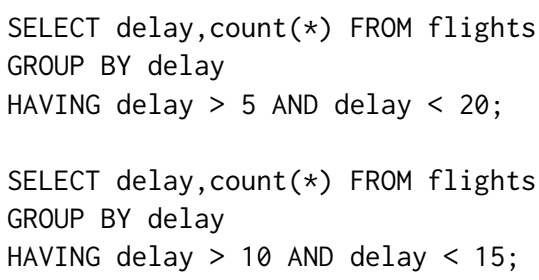

The system parses these two queries into ASTs and finds that the trees differ by the literal nodes representing the numeric bounds in the HAVING clause ${ }^{1}$. Thus, any interface component(s) that can generate two numeric values is a candidate to be mapped to this interaction. We describe possible interface component mappings below.

${ }^{1}$ By analyzing tree differences, the specific syntax doesn't matter to Precision Interfaces. Thus HAVING delay BETWEEN $(5,20)$ would result in the same interfaces. 


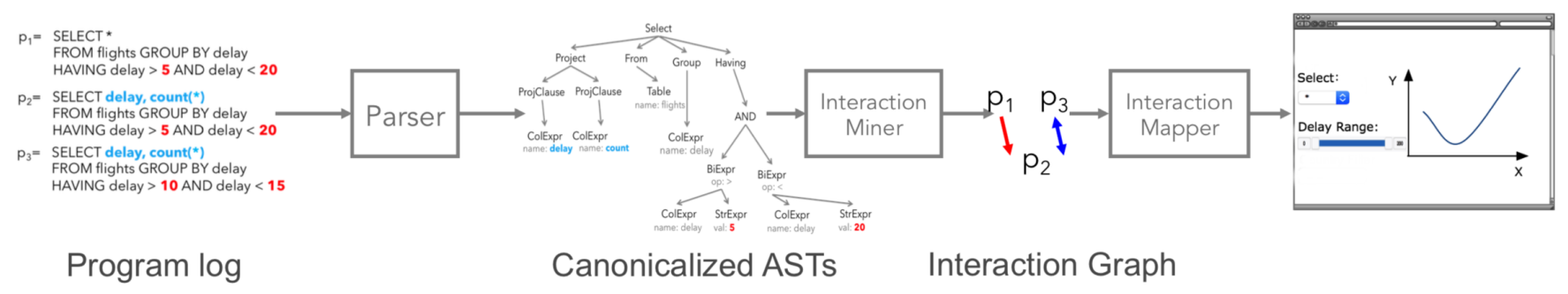

Figure 1: Precision Interfaces parses queries into parse trees, performs tree alignment to generate an interaction graph that is filtered using the PILang domain specific language and whose edges are mapped to interface widgets.

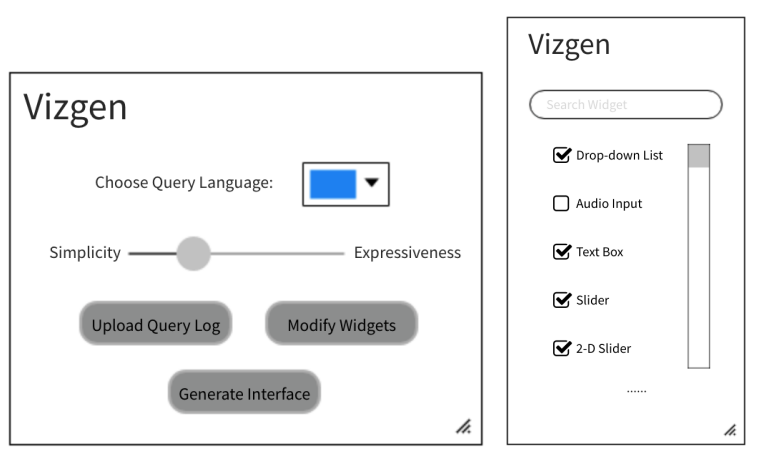

Figure 2: Interface of Precision Interfaces

Precision Interfaces is pre-populated with a set of interface components that the user can filter-for instance, she can choose to exclude text box and check box inputs from consideration. Our demonstration system supports three types of input modalities, that each comes with a different set of components. We describe them below.

Form-based Interfaces Precision Interfaces supports classic form widgets such as buttons, dropdowns, various types of $1 \mathrm{D}$ and $2 \mathrm{D}$ sliders, single and double click, hover, text boxes, radio buttons, and check boxes. Each has a default domain $\Omega$ that can be further restricted through customization. For instance, the domain of the slider is by default $\mathbb{R}$ but can be restricted to have a minimum and maximum value. Similarly, the domain of a dropdown is any set of strings, but can be restricted to a finite set of string literals (e.g., a list of schema attributes, or common values found in the log).

In the example above, the transformation would be mapped to a range slider that lets the user manipulate the minimum and maximum values of the slider. Its state is a numeric pair $\left(v_{\min }, v_{\max }\right)$ and Precision Interfaces will automatically generate the function $f((\min , \max ))$ that maps the pair to the subtree representing the HAVING clause above (Figure 3a). Alternatively, a single button that blindly replaces the first query's AST with the second's is also possible, though it would not generalize to a larger query log.

Gesture-based Interfaces Touch and 3D interfaces rely on contextual gestures to specify interactions $[1,8]$. For example, in a 3D interface (such as Myo band), a user may increase a value by virtually grabbing it and moving her arm to the right. Similarly, in a touch-based interface, it may be expressed by a pinch interaction. Common gesture-based widgets include swipe, tap, zoom, pinch, twist, double tap and press. Each of these can be modeled as transforming some state tuple. For instance, a zoom-pinch interaction may be modeled as scaling the value of a numeric pair. Such an interaction may then be mapped to the example interaction, thus allowing users to interactively scale the flight delay predicate (Figure 3b).

Natural Language Interactions Natural-language Interfaces (NLIs) are a type of computer human interface where linguistic features such as verbs, phrases and clauses act as input controls for creating, selecting and modifying data in software applications. Natural language interfaces such as Google Home and Amazon Echo use command-based interfaces, where the user speaks a command followed by parameters in a Mad-libs fashion; chat-based interfaces provide a command-response interaction where the system asks a question and the user responds.

Precision Interfaces supports simple generation of NLI interfaces as a post-processing step once interaction components have been chosen-the components are then translated into natural language statements. The simplest way is for the developer running Precision Interfaces to simply provide names for each interaction component. For instance, the range slider from the example can be named "delay bounds". Precision Interfaces can then use the slider types and definition to generate the question: "Please provide two numbers to change the Delay Bounds". Similarly, the user can use the command: "Set Delay Bounds to 2 and 10". The specific text presentation can change as long as it elicits a pair of numbers from the user. This creates a conversation to guide users to the query. Thus Precision Interfaces may serve to bootstrap the development of natural language interfaces for new analyses and applications, and serve as a complement to systems such as Ava [9] or NALiR [10].

\section{DEMONSTRATION}

Our demonstration will generate interfaces for the On-Time flight dataset $^{2}$, which contains the departure time, arrival time, airline company, departure location, destination and 86 other attributes for more than 521,000 flights. We chose this data set because the semantics of its attributes is easy to understand and so the quality of the generated interface can be evaluated empirically. We use 2 query logs to demonstrate the effectiveness of our query log based approach. The first query log is manually generated by students

\footnotetext{
${ }^{2}$ https://www.transtats.bts.gov/DL_SelectFields.asp?Table_ID=236
} 


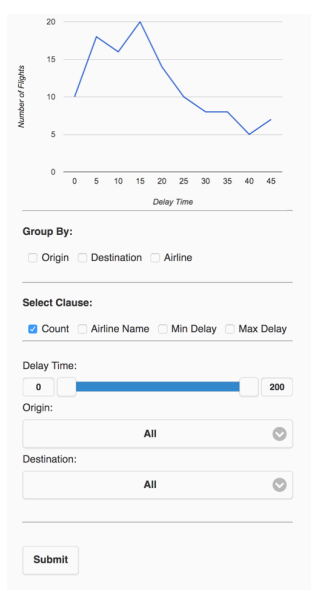

(a) Mouse interface.

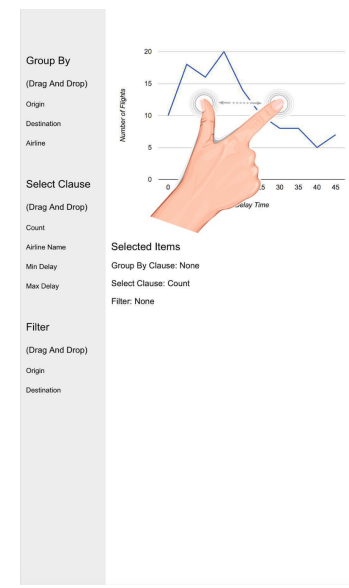

(b) Touch screen interface.

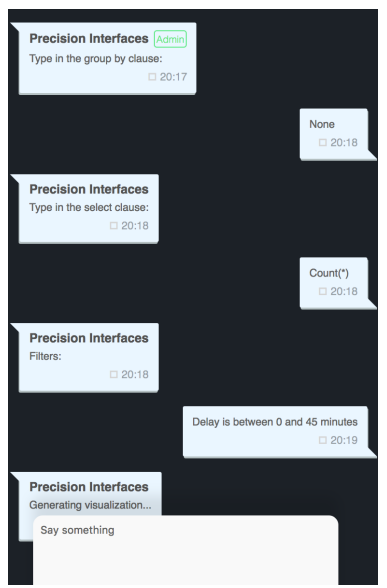

(c) Natural language interface.

Figure 3: Different interfaces generated by Precision Interfaces.

asked to analyze 3 out of 12 predetermined tasks using Tableau and logging the corresponding queries. (e.g., "how delayed are flights to California") and answer one free-form question ("tell us something you find interesting"). The second query log is created using randomly generated queries which do not necessarily provide insightful results. Although the second query log will produce more widgets due to a higher variance between queries, it might not necessarily represent relevant interactions in a succinct manner. On the other hand, the first query log will produce a bespoke interface with less widgets that encapsulates all relevant interactions required by the specific user group.

Generating Interfaces During the first part of the demo, participants will use Precision Interfaces to generate interfaces from query logs. Figure 2 shows the interface, which lets participants choose a query language (currently SQL or SPARQL) and set a trade-off between interface simplicity (less interface components) and expressiveness (can express more queries). Participants will then be able to upload a query log and run the system. If the participants click "Modify Widgets", the right panel appears, which lists the library of interface components and lets them include or exclude components as desired (e.g., select only the gestural interface components). Once the interfaces have been generated, the participants will be able customize them by changing the layout of the interface, tweaking the visualization type, or naming and configuring individual components. A "Publish" button will then compiles the interface into an application.

Using the Generated Interfaces The second part of the demo lets participants use the generated interfaces. Figure 3 shows three interfaces generated with different modalities. Figure 3a only uses form-based widgets, laid out vertically; Figure $3 \mathrm{~b}$ only uses dragging and gestural interactions; Figure $3 \mathrm{c}$ illustrates a naive translation into a chat-based NLI. In all three examples, the user inputs the same information, but through different means. Users will be asked to use the interfaces to examine the number of flights within a delay time range, and the average delay time of flights to New York City and to any destination in California. We will show that the different interfaces are able to perform the same analyses; albeit some interfaces may be easier to use than others.

\section{CONCLUSIONS AND FUTURE WORK}

Our vision is to explore how data-driven approaches can facilitate the creation of tailored interactive analysis interfaces at scale. Precision Interfaces represents an initial approach that is query language agnostic, and can flexibly support a wide variety of existing and future interaction-based user inputs. The demonstration is meant to highlight this vision, and to solicit feedback on the opportunities when studying different modalities and physical interface devices. Acknowledgements This work is supported by NSF grants \#1564049 and \#1527765.

\section{REFERENCES}

[1] J. F. Beltran, Z. Huang, A. Abouzied, and A. Nandi. Don't just swipe left, tell me why: Enhancing gesture-based feedback with reason bins. In IUI, 2017.

[2] S. S. Bhowmick, B. Choi, and C. Dyreson. Data-driven visual graph query interface construction and maintenance: challenges and opportunities. In VLDB, 2016

[3] P. Bille. A survey on tree edit distance and related problems. In TCS, 2005.

[4] M. Bostock, V. Ogievetsky, and J. Heer. $\mathrm{D}^{3}$ data-driven documents. In TVCG, 2011.

[5] T. Gao, M. Dontcheva, E. Adar, Z. Liu, and K. G. Karahalios. Datatone: Managing ambiguity in natural language interfaces for data visualization. In UIST, 2015.

[6] M. Jayapandian and H. Jagadish. Automated creation of a forms-based database query interface. In $V L D B, 2008$.

[7] M. Jayapandian and H. Jagadish. Expressive query specification through form customization. In EDBT, 2008.

[8] L. Jiang and A. Nandi. Snaptoquery: providing interactive feedback during exploratory query specification. In $V L D B, 2015$.

[9] R. J. L. John, N. Potti, and J. M. Patel. Ava: From data to insights through conversations. In $C I D R, 2017$.

[10] F. Li and H. V. Jagadish. Nalir: an interactive natural language interface for querying relational databases. In SIGMOD, 2014.

[11] E. Santos, L. Lins, J. Ahrens, J. Freire, and C. Silva. Vismashup: Streamlining the creation of custom visualization applications. In TVCG, 2009.

[12] A. Satyanarayan, D. Moritz, K. Wongsuphasawat, and J. Heer. Vega-lite: A grammar of interactive graphics. In TVCG, 2017.

[13] H. Zhang, T. Sellam, and E. Wu. Mining precision interfaces from query logs. In arXiv, 2017.

[14] J. Zhang, S. S. Bhowmick, H. H. Nguyen, B. Choi, and F. Zhu. Davinci: Data-driven visual interface construction for subgraph search in graph databases. In ICDE, 2015. 